\title{
Malignant neoplasms of the hematopoietic system in three mutants of Drosopbila melanogaster
}

\author{
by E. GATEFF \\ Biologisches Institut I (Zoologie) Albert-Ludwigs-Universität, \\ 78 Freiburg i. Br., Schänzlestr. 9, W. Germany
}

Two classes of blood cells are found in the larval hemolymph of D. melanogaster; 1. the plasmatocytes with their variants podocytes and lamellocytes and 2. crystal cells (Rizki, 1957, fig. 1). Plasmatocytes are involved in phagocytosis and encapsulation, while crystal cells play a rolle in blood coagulation and melanine production.

The blood cells originate in the hematopoietic organs, the lymph glands, from prohematocytes (small, round cells of Bairati, 1964). The prohematocytes proliferate and provide for both the renewal of the progenitor population and the production of suficient amounts of progeny. A differentiation event gives rise to two types of polygonal cells (terminology of Bairati, 1964) which represent the progenitors for the two classes of blood cells. Polygonal cells with differentiated cytoplasm become plasmatocytes, and polygonal cells with smooth cytoplasm transform into crystal cells (tableau I).

Recently, three larval, recessive-lethal mutations, induced by EMS, were found to cause malignant transformation of the blood-cell progenitors in the lymph glands. The mutant genes are located on the Ist, IInd and IIIrd chromosomes and received the designations lethal (1) malignant blood neoplasm $(l(1) \mathrm{mbn})$, lethal (2) malignant blood neoplasm (l(2) $\mathrm{mbn}$ ) and lethal (3) malignant blood neoplasm (l(3) $\mathrm{mbn}$ ).

A most striking feature of these mutants is the manifold increase of free blood cells in the hemolymph when compared to the wild-type.

This pathological increase of free blood cells is caused by the uncontrolled and excessive proliferation of the progenitors of the phagocytic blood cells in the lymph glands. The number of crystal cells is either normal or reduced. An equally striking characteristics of the mutants are the highly enlarged lymph glands. 
The lymph glands in the mutant $l(l) \mathrm{mbn}$ represent large cell masses, up to 300 times the original size. In contrast to the wild-type larva where the production of new blood cells is strictly controlled, in the mutant this control is absent (tableau I). As a result, a constant stream of plasmatocytes and crystal cells is released from the lymph glands into the hemolymph. The released cells exhibit all main functions

TABLEAU I. - Hematopoiesis in the wild-type larva and the mode of action of the mutant genes $l(1) m b n, l(2) m b n, l(3) m b n$.

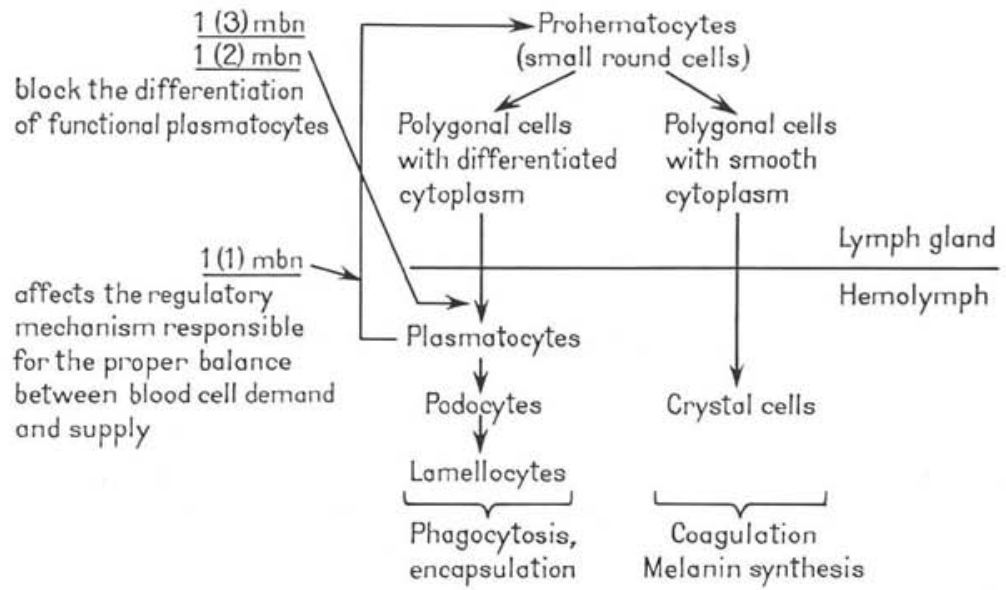

characteristic for normal blood cells, such as, the capacity for phagocytosis, encapsulation and mlanization. These functional abilities, however, are applied in an abnormal fashion. For instance, $l(1) \mathrm{mbn}$ blood cells invade in large numbers the imaginal discs. Furthermore, they have lost the capacity to distinguish «self» from «nonself ». Because of this they encapsulate and melanize large portions of the host's tissues producing lesions which eventually cause its death.

\begin{tabular}{|c|c|c|c|c|}
\hline & wild-type & $l(1) m b n$ & $l(2) m b n$ & $l$ (3) $m b n$ \\
\hline $\begin{array}{c}\text { Number of blood } \\
\text { cells per } \mathrm{mm}^{3} \text { of } \\
\text { hemolymph } \ldots \ldots .\end{array}$ & 1600 & 12500 & 39000 & 225200 \\
\hline
\end{tabular}

A further abnormality of the mutant blood cells is their behavior during transplantation. Wild-type lymph glands, implanted into the abdomens of female flies, do not proliferate. Pieces derived from the $l(1) \mathrm{mbn}$ lymph glands, in contrast, grow excessively, invade the tissues of the host and cause finally its death. The mutant blood cells can be propagated for many transfer-generations in culture in vivo in female flies. 
Thus, the mutation $l(1) \mathrm{mbn}$ causes a lethal, transplantable, malignant neoplasm of the blood cells by affecting the mechanism controlling the supply of new blood cells to the hemolymph (fig. I).

The blood cell neoplasms developing in the lymph glands of the homozygouse $l(2) m b n$ and $l(3) m b n$ mutant larvae are even more severe. The numbers of free blood cells in these mutants exceed manyfold the numbers of free blood cells in the wild-type and the $l$ (l) $m b n$ mutation (fig. 2). In contrast to $l(l) m b n$ in which the neoplastic blood cells show a high degree of differentiation, the $l$ (2) $\mathrm{mbn}$ and $l(3) \mathrm{mbn}$ blood cells are poorely or not at all differentiated. Crystal cells are present in reduced numbers. Similarely to $l(1) m b n$ blood cells the $l(2) m b n$ and $l$ (3) $m b n$ blood cells invade the imaginal discs and destroy their epithelium.

The capacity to melanize differs widely among the three mutants. While $l(1) \mathrm{mbn}$ shows a very strong melanization reaction in situ as well as in culture in vitro, $l(3) \mathrm{mbn}$ exhibits a very weak reaction and $l(2) m b n$ lacks the capacity to melanize. The inability to produce melanin results most probably from the lack of crystal cells in the hemolymph which contain tyrosinase, the enzyme concerned with the conversion of tyrosine to melanin. In vitro cultured $l$ (2) $m b n$ and $l$ (3) $m b n$ blood-cell lines have recently been established.

In conclusion: Three larval, recessive-lethal mutations causing malignant neoplasms of the phagocytic precursor cells have been found. The mutation $l(I) m b n$ affects the control mechanism of blood cell production. Mutations $l(2) m b n$ and $l(3) m b n$ prevent the differentiation of phagocytic blood cells at an early developmental stage, thus, causing the accumulation of large numbers of immature blood cells in the hemolymph. The hematopoietic tumor mutants represent a usefull addition to the allready known true tumor mutants in Drosophila and confirm the prediction of Gateff and Schneiderman (1969) concerning the tissues capable of neoplastic transformation in the larva. Finally, Drosophila hematopoietic mutants may proove usefull in studying the still poorly understood blood-forming events and may be shead some light upon the genetics of the neoplastic transformation of blood cells.

\section{Bibliographie}

Bairati (A.), 1964. - L'ultrastruttura dell' organo dell' emolinfa nella larva di Drosophila melanogaster. Z. Zellforsch. mikr. Anat., 61, 769-802.

GatefF (E.) et Schneiderman (H. A.), 1969. - Neoplasms in mutant and cultured wildtype tissues of Drosophila. Nat. Cancer Inst. Monogr., 31, 365-397.

RIZKI (M. T. M.), 1957. - Alteration in the hemocyte population of Drosophila melano. gaster. J. Morphol., 100, 437-458. 